\title{
Clinical and laboratory findings versus results of renal biopsy: Evaluation the diagnostic value in patients with lupus nephritis
}

\author{
Shila Aghayani ${ }^{1}$, Mohammad Reza Abbasi², Seyed Reza Najafizadeh ${ }^{1}$, Shafieh Movaseghi ${ }^{1}$, \\ Abdolrahman Rostamian, ${ }^{1,3 *}$ \\ ${ }^{1}$ Department of Rheumatology, Imam Khomeini Hospital, School of Medicine, Tehran University of Medical Sciences, \\ Tehran, Iran. ${ }^{2}$ Department of General Surgery, School of Medicine, Nephrology Research Center Imam Khomeini \\ Hospital, Tehran University of Medical Sciences Tehran, Iran. ${ }^{3}$ Rheumatology Research Center, Tehran University of \\ Medical Sciences Tehran, Iran.
}

\begin{abstract}
Kidney involvement is a major cause of death and disability in patients with systemic lupus erythematosus (SLE). Early and accurate determination of the type of involvement is essential in choosing the appropriate treatment for these patients. The current study aimed to determine whether laboratory findings are consistent with kidney biopsy for biopsy classification.

This descriptive analytic cross-sectional study was performed on 17 patients with SLE admitted to rheumatology and nephrology departments. All patients underwent renal biopsy and received appropriate treatment according to the reported pathology. Data analysis was performed using SPSS software version 25. The participants comprised 14 females and 3 males with a mean age of $32.23 \pm 11.12$ years. The findings of this study showed that mean serum C3 and 24-hour urine protein concentrations were significantly different between the four studied groups according to the type of kidney pathology $(p=0.042, p=0.041$; respectively). No significant relationship was found between pathological findings and clinical signs, demographic information, and other laboratory findings $(p>0.05)$. Based on the findings of the present study, it can be concluded that renal biopsy is the most accurate method available for the diagnosis and classification of lupus nephritis. Nonetheless, renal biopsy has limitations including side effects, the need for an experienced pathologist, and suboptimality in some cases; renal biopsy was suboptimal in three cases of the current study. Therefore, noninvasive faster methods with high efficacy should be sought.
\end{abstract}

Keywords: Clinical symptoms, Kidney pathology, Laboratory findings, Lupus nephritis

\section{Introduction}

Diagnosing lupus nephritis is challenging because of the many differences between people in terms of manifestation, clinical course, and severity of the disease. Without careful monitoring of lupus patients, lupus nephritis can lead to ESRD. Therefore, a non-invasive method for diagnosing and monitoring seems to be necessary[1,2].

Serum creatinine and urinary protein levels are helpful in assessing response to treatment, and serum autoantibodies and complements are used along with proteinuria to diagnose lupus nephritis and monitor disease activity. However, these markers cannot differentiate between different stages of lupus nephritis, and there may be inconsistencies between these parameters and histopathology[3, 4].
The current gold standard method for diagnosing lupus nephritis is biopsy and histopathology, although biopsy is an invasive procedure and has complications such as bleeding, venous arterial fistula, and infection. Moreover, it is difficult to repeat[5, 6].

Rapid advances have been made in basic sciences and need to be applied in clinical diagnosis. This study was designed to promote and the use of non-invasive, low-cost, easy methods to diagnose and follow-up on treatment response. The aim of this study was to determine whether laboratory findings are consistent with those of kidney biopsy for biopsy classification.

Personal non-commercial use only. Rheumatology Research Journal. Copyright $($ C 2020. All rights reserved

*Corresponding Author: Dr Abdolrahman Rostamian, Department of Rheumatology, School of Medicine, Tehran University of Medical Sciences, Tehran, Iran, E-mail: arostamian@tums.ac.ir, Phon:+98-912-333-7858.

Received: 27 December 2019; Accepted: 06 February 2020 


\section{Materials and Methods}

\section{Study population}

The study population of this descriptive-analytical cross-sectional study comprised lupus patients with renal involvement who were candidates for kidney biopsy and admitted to the rheumatology or nephrology department of Imam Khomeini Hospital during 2018-2019. According to the one-year census, all patients with lupus nephritis who were candidates for biopsy were included in the study. Inclusion criteria included patients who were diagnosed with SLE according to the 1987 ACR Lupus Diagnostic Criterion and had renal involvement based on U/A and 24hour urine protein results. Exclusion criteria included having a co-morbid history of drug abuse and pregnancy. The protocol of the study was approved by the Ethics committee of Tehran University of Medical Sciences (Code: IR.TUMS.IKHC.REC.1397.243).

\section{Statistical Analysis}

All statistical analyses were performed using SPSS software version 25 (SPSS Inc., Chicago, IL) at both descriptive and analytic levels. The normal distribution of quantitative data was investigated using the KolmogorovSmirnov $\mathrm{Z}$ test, and then one-way analysis of variance (ANOVA) and Kruskal-Wallis tests were employed to compare the four groups with normal and non-normal distribution, respectively. A $p$ value of $<0.05$ was considered as statistically significant.

\section{Results}

In the present study, 20 SLE patients with kidney biopsy initially began the study, but three of them were excluded due to inappropriate biopsy. Ultimately, 3 males and 14 females with a mean \pm SD age of $32.23 \pm 11.12$ years completed the study. The demographic information of the patients, such as sex, age, laboratory data such as AntidsDNA, C3, C4, HTN, WBC, Hb, PLT, Cr, ESR, CRP, and 24-hour urine protein as well as kidney biopsy results were collected. Next, the relationship between laboratory and demographic findings with pathology results was assessed.

The percentages of males and females were $17.65 \%$ and $82.35 \%$, respectively. The frequency of edema and HTN positivity in the studied patients was $35.29 \%$ and $29.41 \%$, respectively. According to Table 1, the mean and standard deviation of Anti-dsDNA, C3, C4, WBC, Hb, PLT, Cr, ESR, CRP, and urinary protein 24 are shown.

The results showed that sex, age, edema, and HTN were not significantly associated with the pathological findings. As shown in Table 2, there was no significant difference between the groups in the laboratory findings of lupus activity. It should be noted, however, that there was a significant relationship between serum C3 level and 24hour urinary protein pathological findings.

Table 1. Laboratory findings of the subjects

\begin{tabular}{cc}
\hline Parameters & Mean \pm SD/ Percentage \\
Creatine & $1.8 \pm 1.12$ \\
Anti-dsDNA & $280.85 \pm 209.317$ \\
C3 & $57.82 \pm 28.66$ \\
C4 & $17.03 \pm 19.39$ \\
Urine WBC & $5.56 \pm 4.67$ \\
Urine RBC & $13.64 \pm 8.16$ \\
Urine protein & $1.35 \pm 1$ \\
24 hours urine protein & $1408.94 \pm 828.34$ \\
& II: 1 \\
Nephritis stage & III: 3 \\
HLT & IV: 11 \\
Hb & V: 2 \\
HBC & $3424.7 \pm 3071.8$ \\
\hline
\end{tabular}




\begin{tabular}{cc}
\hline Parameters & Mean \pm SD $/$ Percentage \\
\hline CRP & $9.94 \pm 10.63$ \\
ESR & $63.11 \pm 40.11$ \\
\hline
\end{tabular}

Table 2. Evaluation of the relationship between renal biopsy results and laboratory findings of lupus activity.

\begin{tabular}{cccccc}
\hline Stage & Creatinine & 24 hours urine protein & Anti-dsDNA & C3 & C4 \\
\hline II & 0.7 & 792 & 180 & 68 & 9.2 \\
III & $0.9 \pm 1.96$ & $896.55 \pm 1388.66$ & $97.43 \pm 101.06$ & $15.71 \pm 55$ & $4.04 \pm 9.33$ \\
IV & $1.21 \pm 2.03$ & $553.87 \pm 1207.09$ & $206.47 \pm 363.63$ & $25.29 \pm 48.63$ & $20.73 \pm 16.58$ \\
V & $0.28 \pm 0.8$ & $1128.54 \pm 2858$ & $191.41 \pm 145.65$ & $16.24 \pm 107.5$ & $28.28 \pm 35$ \\
Total & $1.12 \pm 1.8$ & $828.34 \pm 1408.94$ & $209.317 \pm 280.85$ & $28.66 \pm 57.82$ & $19.39 \pm 17.0 .3$ \\
P value & 0.412 & 0.041 & 0.168 & 0.042 & 0.48
\end{tabular}

Table 3. Evaluation of the relationship between renal biopsy results and laboratory findings of lupus activity.

\begin{tabular}{cccc}
\hline Stage & ESR & CRP & PLT \\
\hline II & 125 & 12 & 108000 \\
III & $16.82 \pm 34$ & $6.24 \pm 7$ & $59 / 245395 \pm 230000$ \\
IV & $37.26 \pm 72.54$ & $12.35 \pm 12$ & $55 / 164999 \pm 36 / 227636$ \\
V & $29.69 \pm 24$ & $1.41 \pm 2$ & $98 / 19798 \pm 343000$ \\
Total & $40.11 \pm 63.11$ & $10.63 \pm 9.94$ & $6 / 164545 \pm 23 / 234588$ \\
P value & 0.073 & 0.658 & 0.727 \\
\hline
\end{tabular}

\section{Discussion}

The type of renal involvement in SLE is an important determinant of prognosis in these patients. It is essential to determine early the correct type of involvement so as to choose the appropriate treatment for these patients. The most correct way to determine the type of kidney lesion is through pathological examination of kidney tissue[7]. Because of its invasiveness, related complications, and a lack of access to necessary facilities (experienced pathologists, equipment, etc.), use of this method is not possible for all physicians[8]. The present study was performed to determine the relationship between appropriate clinical and paraclinical criteria by determining the type of renal involvement in 14 female and 3 male SLE patients with renal involvement. The findings of this study showed that the means of $\mathrm{C} 3$ and 24-hour urinary protein were significantly different among the 4 studied groups. No significant relationship was found between renal pathology findings and clinical signs, demographic information, or other laboratory findings. Renal biopsy was suboptimal in three cases, indicating this is one of the limitations of pathology assessments of lupus nephritis patients.

Saadati et al. [9] investigated the relationship between clinical symptoms and renal pathology in patients with systemic lupus erythematosus. Of the 34 patients studied, 32 were female and 2 were male. The mean age of patients at the time of diagnosis was $25.12 \pm 12.05$ years. All patients had kidney involvement at the time of referral. The most common pathological renal lesion in these patients was diffuse proliferative glomerulonephritis. Class 4 lupus nephritis was the most important factor in determining the prognosis. The sex ratio in the present study was similar to that in the study of Saadati et al., however unlike the study of Saadati et al., the current study did not examine the prognoses of patients.

Ebadi et al.[10] evaluated the epidemiology of lupus nephritis in 40 patients admitted to Imam Khomeini Hospital. The age range of patients in their study was between $12-45$ years with a mean age of 21.5 years, and the maximum frequency in the second decade of life was $48.6 \%$. In their study, $86.5 \%$ of patients were female and $13.5 \%$ were male. Butterfly rash and fever were the most 
common clinical symptoms with $62.5 \%$ and $57.5 \%$, respectively. The most common pathology and symptoms of renal involvement were class IV with $37.8 \%$ and hematuria with $88.9 \%$, respectively, and. $97.1 \%$ of patients had renal involvement. Pulse therapy with corticosteroids and cyclophosphamide was effective in $50 \%$ of cases in pathology IV in preventing chronic renal failure. $100 \%$ of patients with class III lupus nephritis had poor prognosis. In the present study, contrary to the results of Ebadi et al.'s study, patients with pathology class 5 had higher levels of $\mathrm{C} 3$ and 24-hour urinary protein. In line with the results of Ebadi et al., the most common type of pathology in the present study was class 4 .

Sufivand et al. (no published data) evaluated the diagnostic value of laboratory and serological clinical findings of 30 patients with lupus nephritis in comparison with the biopsy results of all patients in need of biopsy in rheumatology and nephrology wards of Imam Hospital. The results of their study showed that $5,8,10$, and 7 cases had grade 3, grade 3 and 4, grade 4, and grade 5 biopsy results, respectively. The relationship between age, proteinuria, ESR, and biopsy result was significant. The results of this study contradict the findings of the present study which showed no significant relationship between age, ESR, and biopsy results. The study has a potential limitation in its sample size. Unfortunately, despite meeting the criteria for renal biopsy, some patients were dissatisfied for renal biopsy.

\section{Conclusion}

Based on the findings of the present study, it can be concluded that renal biopsy is the most accurate available method for the diagnosis and classification of lupus nephritis. However, because of the limitations of renal biopsy, which include side effects, the need for an experienced pathologist, and suboptimality in some cases (three cases in the current study), noninvasive, faster methods with high efficacy should be sought. Repetition of biopsy is difficult, and this classification can be done with laboratory findings. Although it was shown that there is a significant relationship between serum C3 levels and 24hour urinary protein with pathological findings, more studies are required to confirm the findings of the current study.

\section{Acknowledgments}

None.

\section{Conflict of interest}

The authors declare no conflicts of interest.

\section{References}

1. Bobart SA, De Vriese AS, Pawar AS, Zand L, Sethi S, Giesen C. et al. Noninvasive diagnosis of primary membranous nephropathy using phospholipase A2 receptor antibodies. Kidney Int 2019; 95(2):429-38. doi: 10.1016/j.kint.2018.10.021.

2. Anders HJ, Saxena R, Zhao MH, Parodis I, Salmon JE, Mohan C. Lupus nephritis. Nat Rev Dis Primers 2020; 6(1):7. doi: 10.1038/s41572-019-0141-9.

3. Reyes-Thomas J, Blanco I, Putterman C. Urinary biomarkers in lupus nephritis. Clin Rev Allergy Immunol 2011; 40(3):138-50. doi: 10.1007/s12016-010-8197-z.

4. Kudose S, Santoriello D, Bomback AS, Stokes MB, D'Agati VD, Markowitz GS. Sensitivity and Specificity of Pathologic Findings to Diagnose Lupus Nephritis. Clin J Am Soc Nephrol 2019; 14(11):1605-15. doi: 10.2215/cjn.01570219.

5. Schwartz N, Goilav B, Putterman C. The pathogenesis, diagnosis and treatment of lupus nephritis. Curr Opin Rheumatol 2014; 26(5):502-09. doi: 10.1097/bor.0000000000000089.
6. Davidson A, Aranow C, Mackay M. Lupus nephritis: challenges and progress. Curr Opin Rheumatol 2019; 31(6):682-88. doi: 10.1097/bor.0000000000000642.

7. Moroni G, Depetri F, Ponticelli C. Lupus nephritis: When and how often to biopsy and what does it mean? $\boldsymbol{J}$ Autoimmun 2016; 74:27-40. doi: 10.1016/j.jaut.2016.06.006.

8. Yim T, Kim SU, Park S, Lim JH, Jung HY, Cho JH. et al Patterns in renal diseases diagnosed by kidney biopsy: A single-center experience. Kidney Res Clin Pract 2020; 39(1):60-69. doi: 10.23876/j.krcp.19.077.

9. Sadati N, Hami M, Aghdam-ataolah B, Naghibzadeh B. Evaluation of the relationship between clinical symptoms and renal pathology in patients with systemic lupus erythematosus.

10. Ebadi S, Zamani B, Soleimani A, Tamadon M, Arbabi M. Epidemiologic study of 40 patients with lupus nephritis in Imam Khomeini. Hormozgan Medical Journal 2006; 10(3):231-36. 